Revista PSICOLOGIA, 2017, Vol. 31 (1), 59-68. doi: 10.17575/rpsicol.v31i1.1285

\title{
Normas de categorias ad hoc para língua portuguesa
}

\author{
Jeronimo Cassol Soro ${ }^{1} \&$ Mário Augusto Boto Ferreira ${ }^{1}$ \\ ${ }^{1}$ CICPSI, Faculdade de Psicologia, Universidade de Lisboa
}

\begin{abstract}
Resumo: A investigação sobre organizações de representações mentais tem vindo a revelar desenvolvimentos teóricos muito relevantes ao focar-se na flexibilidade destas estruturas e na sua dependência do contexto. Categorias ad hoc são particularmente úteis para esta investigação por possuírem estruturas hierárquicas semelhantes a categorias taxonómicas, mas sendo simultaneamente, e por definição, altamente dependentes do contexto e de objetivos. 0 presente estudo fornece medidas normativas Portuguesas para 63 listas de exemplares de diferentes categorias. Dois tipos de categorias ad hoc são apresentados: 35 inter-categóricas (compostas por exemplares provenientes de diferentes categorias taxonómicas) e 28 intra-categóricas (compostas por exemplares de uma mesma categoria taxonómica). São ainda incluídas normas relativamente a 10 categorias taxonómicas de onde as categorias ad hoc intra-categóricas foram derivadas. As listas de exemplares categóricos são apresentadas com medidas de frequência de produção, frequência de primeira produção, e posição média da ordem de produção.
\end{abstract}

\section{Palavras-chave: Categorias ad hoc; Normas; Frequência de produção; Categorização.}

Portuguese norms for ad hoc categories: Scientific investigation on the organization of mental representations of categories has been showing, over the years, important new developments by focusing on the flexibility of these knowledge structures and its context dependency. Ad hoc categories are of particular use in this research domain because although their structures are hierarchically organized (as taxonomic categories are) they are, by definition, highly dependent on context and goals. Our study presents Portuguese norms for 63 lists of exemplars from different ad hoc categories. Two types of ad hoc categories are presented: 35 inter-categorical (composed of exemplars from different taxonomic categories), and 18 intra-categorical (composed of exemplars from the same taxonomic category). The present work further includes norms for 10 taxonomic categories, from which the intra-categorical ad hoc lists are derived. The categorical exemplars lists are presented with measures of production frequency, frequency of first production and production order average rank.

Keywords: Ad hoc category; Norms; Production frequency; Categorization.

As normas para listas de exemplares pertencentes a categorias taxonómicas (e.g., Frutas, Animais, Desportos) são um valioso material para investigação em psicologia cognitiva uma vez que nos fornecem uma medida de força associativa entre conceitos através de diferentes níveis hierárquicos de informação. Esta informação assume um papel crucial na investigação ao permitir o estudo de processos mentais específicos manipulando ou controlando para a força associativa dos estímulos (exemplares) usados. Estas estruturas associativas ocorrem também para itens de categorias que não são taxonómicas, e que, por serem tão específicas, dificilmente possuem associações estáveis na memória de longo prazo. Um exemplo disto são as categorias $a d$ hoc. Ou seja, categorias criadas espontânea e pontualmente em função de objetivos específicos relevantes para a situação onde o indivíduo se encontra (Barsalou, 1983). Categorias ad hoc têm tido crescente relevância para a psicologia cognitiva por se tratarem de uma excelente ferramenta para a investigação sobre representações situadas de conceitos, que possuem características flexíveis e dinâmicas. No entanto, as normas de listas ad hoc são escassas, o que justifica o objetivo do presente trabalho: apresentar normas para um número relativamente alargado de categorias ad hoc que permitam e facilitem a investigação em língua portuguesa nesta área.

No sentido de oferecer um enquadramento teórico para a apresentação destas normas começaremos por discutir os fatores que tipicamente são considerados na determinação da estrutura hierárquica das categorias. Serão introduzidas e explicadas duas medidas frequentemente utilizadas para aceder às estruturas hierárquicas e os dois principais processos de categorização.

${ }^{1}$ Endereço para correspondência: Jerónimo Soro, Faculdade de Psicologia, Universidade de Lisboa, Alameda da Universidade, 1649-013 Lisboa, Portugal. E-mail: jeronimosoro@campus.ul.pt 
Depois caracterizaremos melhor as categorias ad hoc, nomeadamente a sua estrutura hierárquica, assim como a sua origem decorrente de um processo de categorização diferente do envolvido em categorias taxonómicas.

A partir deste enquadramento inicial, desenvolveremos a noção de categoria ad hoc enquanto um caso especial de categorias derivadas de objetivos (Barsalou, 1991), discutiremos brevemente a utilidade de categorias ad hoc para estudos sobre representações flexíveis de conceitos e, por fim, descreveremos o método usado para obtenção de normas de frequência de produção de exemplares de categorias ad hoc.

\section{ESTRUTURAS HIERÁRQUICAS DE CATEGORIAS}

Ao encontrar um pássaro nunca antes visto podemos, contudo, deduzir uma série de características suas pelo simples facto de o incluirmos na categoria "pássaros". Por exemplo, "voa", "põe ovos", "tem sangue quente". Desta forma, a categorização de elementos encontrados na realidade permite-nos estabelecer uma mesma identidade para itens que existem de forma independente, e assim deduzir as características existentes num a partir do outro. Neste sentido, a classificação e identificação de categorias traduz-se em evidentes ganhos de eficiência no processamento cognitivo da informação (e.g., melhor desempenho em recordação livre para grupos de itens pertencentes à mesma categoria; Puff, 1970) e no estabelecimento da melhor forma de interagir com cada elemento encontrado na realidade, o que não seria possível caso tratássemos cada novo elemento encontrado como totalmente independente e não relacionado com a categoria a que pertence. Em suma, não é preciso identificar e verificar as características de um elemento sempre que o encontramos, uma vez que estas são induzidas da categoria na qual este elemento foi incluído (Rips, 1975).

Este processo de categorização não é, no entanto, reducionista ao ponto de todos os exemplares de uma categoria terem igual saliência ou representatividade dentro da mesma. Somos sensíveis à variedade dos exemplares de uma categoria e isto traduz-se em estruturas hierárquicas, com exemplares mais fortemente associados ao conceito da categoria e outros menos (Rosch, 1973). Por exemplo, mesmo que consideremos "pardal", "pinguim" e "avestruz" como exemplares da mesma categoria (Aves), "pardal" salienta-se por ser percebido como um exemplar de Ave claramente mais típico do que "pinguim" ou "avestruz".

\section{MEDIDAS DE ESTRUTURAS HIERÁRQUICAS}

Embora se possa recorrer a várias medidas para aceder às estruturas hierárquicas de categorias como a tendência central, ideais (Barsalou, 1985) ou familiaridade (Casey, 1992; Hampton \& Gardiner, 1983), as duas medidas mais frequentemente utilizadas são a tipicidade e a frequência de produção (Mervis, Catlin \& Rosch, 1976). A tipicidade é normalmente obtida apresentando exemplares de uma categoria e pedindo aos sujeitos que os ordenem do mais ao menos típico ou que avaliem numa escala o grau em que cada item é um bom exemplar da categoria em questão (ou o quanto este está próximo do conceito da categoria). A frequência de produção obtém-se fornecendo aos sujeitos o nome de uma categoria e pedindo que listem exemplares desta categoria. Obtêm-se assim medidas da frequência com que os exemplares foram indicados pelos sujeitos como pertencentes à categoria em questão.

As normas de frequência de produção foram uma das primeiras formas de aceder (e evidenciar a existência de) estruturas hierárquicas de organização categórica. Originalmente, esta medida surgiu no âmbito das teorias associativistas gerais de organização semântica hierárquica baseadas na existência de nódulos conceptuais e de ligações associativas de diferentes intensidades entre eles (Collins \& Loftus, 1975). A medida de frequência de produção indicaria assim a força associativa entre a categoria e os exemplares que a compõem. Aqueles com mais força associativa seriam exemplares com mais probabilidade de serem produzidos a partir da categoria. A relevância desta medida para a compreensão das estruturas categóricas fica evidente em investigações que mostram a relação entre a frequência de produção e os efeitos ligados à representação e processamento de categorias: exemplares com maior frequência de produção a) são mais rapidamente identificados como membros da categoria (Loftus, 1973; Wilkins, 1971); b) têm mais tendência a serem recordados de forma agrupada (i.e., surgem contíguos na ordem de recordação livre ao contrário de exemplares com baixa frequência de produção que tendem a surgir intercalados com exemplares de outras categorias; Bousfield, Cohen, \& Whitmarsch, 1958); c) são falsamente reconhecidos mais frequentemente (Smith, Ward, Tindell, Sifonis, \& Wilkenfeld, 2000); e d) servem mais vezes como ponto de partida para geração de ideias criativas (Ward \& Wickes, 2009).

A medida de tipicidade surge na literatura científica com o objetivo de investigar a estrutura hierárquica de categorias taxonómicas e assim refutar empiricamente a perspetiva Aristotélica de que a inclusão de exemplares numa categoria se faria pela definição de um conjunto de atributos necessários e suficientes (Rosch, 1973). Com efeito, em oposição a esta ideia, Rosch e Mervis (1975) mostraram existir uma correlação positiva entre a tipicidade de um exemplar e a quantidade de atributos que este exemplar 
compartilha com outros exemplares da mesma categoria (medida a que se chamou "semelhança de família" - "family resemblance" no original), assim como uma correlação negativa entre tipicidade e os atributos compartilhados com exemplares de outras categorias. Ou seja, subjacente à tipicidade está a ideia de que as estruturas das categorias se organizam hierarquicamente por comparação dos exemplares a um protótipo que é composto por atributos ideais da categoria (ou atributos que correspondem à média dos atributos dos exemplares da categoria). Quanto mais similar a este protótipo forem os exemplares, mais típicos serão. A investigação subsequente mostrou ainda que exemplares mais típicos são mais rapidamente identificados como membros de uma categoria, (McCloskey \& Glucksberg, 1978); são mais rapidamente aprendidos no contexto de uma nova categoria (Rosch, Simpson \& Miller, 1976); e que características atribuídas a exemplares típicos são mais prováveis de serem inferidas em novos exemplares (Rips, 1975).

Apesar das diferenças entre os fatores relacionados com medidas de tipicidade e frequência de produção, e apesar de alguma variação nas correlações encontradas entre estas medidas e alguns processos cognitivos, elas tendem a surgir consistentemente correlacionadas entre si (Mervis, Catlin, \& Rosch, 1976). Com efeito, é difícil criar listas de categorias que dissociem as duas medidas, o que dificulta observar com clareza qual das duas medidas melhor capta a estrutura hierárquica duma categoria ${ }^{2}$.

Em suma, embora os esforços de diferenciação entre as duas medidas possuam interesse teórico no sentido de melhor compreender a estruturação de categorias na memória de longo prazo, a forte correlação normalmente encontrada faz com que nenhuma assuma precedência enquanto forma de aceder à estrutura hierárquica duma categoria.

\section{PROCESSOS DE CATEGORIZAÇÃo}

A criação e estruturação de representações mentais de categorias pode ocorrer através de dois processos principais, que não são mutuamente exclusivos: aprendizagem de exemplares e combinação conceptual (Barsalou, 1991). A categorização por aprendizagem de exemplares dá-se através da extração das características percebidas de exemplares encontrados no ambiente e da integração destas características como fazendo parte de uma nova categoria (Medin \& Schaffer, 1978; Rosch \& Mervis, 1975). Por exemplo, ao encontrar um novo tipo de comida, extraímos as suas características (e.g., aspeto, sabor, ingredientes de base) e integramo-las na representação cognitiva de uma nova categoria. A categorização por combinação conceptual dá-se através da manipulação de representações mentais já existentes para criação de conceitos novos e normalmente mais complexos (Murphy, 1988). Este processo pode, inclusive, prescindir totalmente do encontro com entidades no ambiente. Assim, por exemplo, podemos manipular o conhecimento sobre efeitos da poluição e fenómenos naturais para derivar novas categorias como "chuvas ácidas", "lixo oceânico", etc.

A categorização por aprendizagem de exemplares pode ser entendida como o processo predominante na origem de categorias taxonómicas (e.g., "frutas”, “animais”,"profissões”, “desportos”). Esta categorias foram largamente utilizadas em estudos iniciais sobre processos de categorização, provavelmente por serem compostas de conceitos simples, cujos exemplares são encontrados com frequência na realidade e são reconhecidos pela grande maioria das pessoas com facilidade. A categorização por combinação conceptual, por sua vez, favorece a criação de categorias mais complexas e abstratas, que podem ser comuns (e.g., "animais pesados"), incomuns (e.g., "escadas confortáveis") ou até imaginários (e.g., "flores que falam"). As categorias taxonómicas servem o propósito de organizar a realidade à nossa volta e facilita-nos a capacidade de inferir características em elementos novos. As combinações conceptuais, por seu lado, servem propósitos mais variados (Barsalou, 1991; Wisniewski, 1997), como a implementação de uma organização mais sofisticada e específica dos elementos da nossa realidade (e.g., casas para animais), a produção de um conhecimento idealizado não existente (e.g., vida alienígena), ou uma organização complexa voltada para a realização de um objetivo (e.g., alimentos com poucas calorias). Nesta linha, um tipo de categoria que se baseia no processamento de combinação conceptual são as categorias ad hoc, também chamadas goal-derived ou categorias guiadas por objetivos (Barsalou, 1983; 1991).

\footnotetext{
${ }^{2}$ Hampton (1997, Experimento 2) faz uma tentativa de dissociar tipicidade e frequência de produção numa manipulação que afeta a forma com que a tarefa é processada, favorecendo um processamento mais orientado para a tipicidade ou para a frequência de produção dos exemplares de categorias (verificação de semelhanças de características e a ativação associativa de conceitos, respetivamente). Apesar dos resultados mostrarem efeitos diferentes para cada condição da manipulação, os dados parecem indicar o uso estratégico dos dois tipos de processamento por parte dos participantes. Outro exemplo de dissociação das medidas, este talvez mais claro, foi obtido em Keller e Kellas (1978) onde se observou diminuição da interferência pró-ativa (release of proactive interference) para categorias quando os itens passavam de típicos para atípicos, mas não quando os itens passavam de menos para mais frequentemente produzidos.
} 


\section{CATEGORIAS AD HOC COMO UM CASO PARTICULAR DE CATEGORIAS GUIADAS POR OBJETIVOS}

As categorias guiadas por objetivos (goal-derived categories) diferenciam-se das taxonómicas justamente por terem como conceito organizador a função de alcançar um objetivo. Dentro das categorias guiadas por objetivos, as que apresentam objetivos de natureza mais efêmera e pontual são as categorias ad hoc. Estas são criadas como forma de responder a fins específicos e transitórios que surgem no nosso dia a dia. A sua natureza ad hoc implica que não possuem representações estáveis na memória de longo prazo. Um exemplo de categoria ad hoc é, "objetos a salvar de uma casa em chamas", uma categoria que será seguramente criada no momento em que encontremos a nossa casa a arder, mas que a maioria de nós, desejavelmente, nunca terá que gerar. Note-se, contudo, que o uso continuado de uma categoria ad hoc (o que pode acontecer, no caso do exemplo acima, àqueles de entre nós que resolverem juntar-se aos bombeiros voluntários) levará a mais estabilidade na sua representação mental, perdendo progressivamente a sua natureza ad hoc.

A falta de estabilidade das representações mentais de categorias ad hoc é evidente na menor força associativa entre os exemplares e a categoria, em comparação com categorias taxonómicas (Barsalou, 1983). Outra característica em que as categorias ad hoc e taxonómicas diferem, é o facto dos exemplares que as compõem geralmente serem provenientes de diferentes categorias taxonómicas. Se pensarmos em exemplares para a categoria ad hoc já mencionada ("objetos a salvar de uma casa em chamas"), poderíamos ter como exemplares "pessoas", "animais", "dinheiro" e "computador". Isto implica que as categorias ad hoc não têm, à partida, uma estrutura correlacional. Por outras palavras, os exemplares de uma categoria ad hoc não possuem necessariamente atributos que coocorram com frequência como no caso das categorias taxonómicas (e.g., "possui penas" e "voa" são atributos que coocorrem com frequência na categoria "aves").

Apesar das diferenças que as categorias ad hoc têm em relação às taxonómicas, e apesar de sua efemeridade, elas são consideradas categorias pelo facto de possuírem estrutura interna hierárquica. Com efeito, Barsalou $(1983,1985)$ mostrou que quando instruídas a gerar membros de categorias ad hoc, as pessoas produzem exemplares com diferentes graus de frequência de produção. Para além disto, os julgamentos de tipicidade mostram consistência inter-participante, existindo correlações significativas entre julgamentos de tipicidade e frequência de produção. Todas estas evidências apontam para a possibilidade e necessidade de obtenção de normas de exemplares para categorias ad hoc.

\section{NORMAS DE CATEGORIAS AD HOC}

0 estudo da estrutura e processos cognitivos subjacentes às categorias ad hoc é, por si só, uma área de investigação importante em psicologia cognitiva (e.g., Barsalou, 1991) dada a sua relevância na compreensão da flexibilidade das representações mentais que desenvolvemos do mundo. Com efeito, investigação recente tem vindo a dar cada vez mais ênfase à natureza dinâmica do conhecimento humano (e.g., Barsalou, 1999; Casasanto \& Lupyan, 2015). Por outro lado, normas de categorias ad hoc podem servir como material para a investigação em psicologia que envolva a manipulação de contextos e/ou objetivos do quotidiano das pessoas. Com conhecimento dos exemplares que tendem a ser produzidos por estas categorias e da forma como estes se organizam, torna-se possível observar diferenças na representação (tanto individual quanto em grupo) destes exemplares em função da maneira como os contextos e/ou objetivos subjacentes aos temas ad hoc são manipulados (e.g., explicitamente apresentados ou apenas tacitamente inferidos), assim como o seu impacto em diferentes processos cognitivos, como memória, atenção e julgamento. Contudo, existe uma escassez de normas para categorias ad hoc que limita, ou pelo menos dificulta, a investigação experimental nesta área. Com efeito, as categorias ad hoc estão ausentes das largamente referenciadas normas de frequência de produção de Battig e Montague (1969). Na atualização destas normas por Van Overschelde, Rawson e Dunlosky (2004) foram incluídas apenas sete categorias ad hoc o que, embora aponte para um aumento do reconhecimento do potencial experimental destas categorias, é ainda um número manifestamente insuficiente.

0 objetivo deste artigo é colmatar, ainda que parcialmente, esta insuficiência, apresentando normas portuguesas de frequência de produção para 63 categorias ad hoc. Destas 63 listas, 14 (cerca de $22.2 \%$ ) foram originadas em (ou inspiradas por) publicações anteriores (Barsalou, 1983, 1985; ValeéTorangeau, Anthony, \& Austin, 1998); as demais foram criadas pelo primeiro autor. As categorias foram divididas em dois grandes grupos: Inter- e Intra-categóricas. As categorias ad hoc inter-categóricas são as que não possuem estrutura correlacional (i.e., são compostas por exemplares que compartilham poucas características que coocorrem na realidade). As intra-categoriais, por sua vez, possuem estrutura correlacional uma vez que todos os seus exemplares pertencem a uma categoria taxonómica à qual foi associado um objetivo específico que reorganiza a sua estrutura hierárquica de frequência de produção (e.g.,"Frutas que servem para atirar aos outros"). Esta diferenciação não aparece, tanto quanto sabemos, 
em referências anteriores às listas ad hoc. Contudo, ela faz sentido, pois tem como base uma característica importante para estas categorias (a presença ou não de estrutura correlacional). As categorias ad hoc intra-categóricas possibilitam assim experimentação com um material que contém associações semânticas em função da sua taxonomia, mas que tem simultaneamente o potencial de sofrer uma categorização contextual voltada a objectivos. Neste sentido, para facilitar uma comparação entre estruturas ad hoc intra-categóricas e as respetivas categorias taxonómicas, disponibilizamos também as versões taxonómicas das categorias ad hoc intra-categóricas aqui expostas.

\section{MÉTODO}

\section{Participantes}

Quatrocentos e vinte e cinco alunos de psicologia da Universidade de Lisboa participaram no estudo em troca de créditos de participação.

\section{Material}

Foram recolhidas produções de exemplares para 63 categorias ad hoc. Destas, 35 são inter-categóricas (sem estrutura correlacional) e 28 são intra-categóricas (compostas por um sub-conjunto de exemplares de uma mesma categoria taxonómica). Foram também recolhidas produções de exemplares para 10 categorias taxonómicas de onde as categorias ad hoc intra-categóricas são originadas. 0 número de participantes que gerou exemplares para cada categoria variou entre 20 e 69.

Na Tabela 1 apresentam-se as listas, conjuntamente com o seu artigo de origem (nos casos de categorias previamente existentes em língua inglesa), a quantidade de participantes respondentes e a potência de produção. A potência de produção refere-se à quantidade média de itens produzido por participante em cada lista, sendo obtida através da divisão do número total de produções pelo número total de participantes que produziram exemplares para a lista em questão. A lista com menos produções individuais ("Instrumentos musicais com duas ou mais partes fisicamente separadas") apresenta uma média de 1.77 produções e a lista com mais produções ("Roupas") apresenta uma média de 8.51 produções individuais.

Tabela 1. Tipo de categorias e categorias usadas no estudo; número de participantes (N) que produziram exemplares para cada categoria; e potência de produção para cada lista

\begin{tabular}{|c|c|c|}
\hline & $\mathbf{N}$ & Potência \\
\hline \multicolumn{3}{|l|}{ Ad hoc inter-categoriais } \\
\hline Coisas que podem ser usadas para retirar um gato de cima de uma árvore & 48 & 3.92 \\
\hline Coisas inflamáveis (a) & 41 & 4.80 \\
\hline Presentes de aniversário (b) & 41 & 7.00 \\
\hline Coisas que se levam para acampamento (b) & 41 & 6.95 \\
\hline Coisas que se levam numa bagagem de mão (a) & 41 & 6.07 \\
\hline Coisas que podem ser compradas na Feira da Ladra (c) & 40 & 5.70 \\
\hline Coisas encontradas nos "perdidos e achados" & 41 & 5.68 \\
\hline Coisas para se ter num abrigo nuclear & 40 & 4.73 \\
\hline Objetos que podem servir como "recordações" & 41 & 5.27 \\
\hline Coisas que podem ser usadas como superfície de apoio para escrever & 41 & 5.46 \\
\hline Objetos que podem ser usados para chamar a atenção de uma pessoa num prédio distante durante o dia & 40 & 5.13 \\
\hline Objetos que podem ser usados para amortecer a queda de um pequeno bibelot & 40 & 5.13 \\
\hline Coisas pesadas que podem ser compradas numa mercearia & 39 & 5.44 \\
\hline Objetos que podem ser utilizados para esmagar uma laranja com casca & 39 & 4.90 \\
\hline Coisas baratas e servidas rapidamente que podem ser pedidas num restaurante & 38 & 5.71 \\
\hline Coisas que podem assustar uma vaca & 38 & 5.05 \\
\hline Coisas perigosas que os bebés correm o risco de pôr na boca & 38 & 5.32 \\
\hline Coisas que podem ser usadas para impedir uma porta de fechar & 45 & 5.62 \\
\hline Objetos que podem ser usados como apoio para uma panela quente & 45 & 5.18 \\
\hline Objetos que podem ser usados para limpar sujidade debaixo de uma unha & 45 & 5.40 \\
\hline Objetos que podem servir como brinquedo de roer a um cão & 45 & 5.36 \\
\hline Objetos que podem ser usados como baquetas para fingir que se toca bateria & 44 & 5.23 \\
\hline Ações facilmente identificáveis através de mímica & 20 & 6.50 \\
\hline Objetos que podem ser usados para proteger o rosto do vento & 44 & 5.23 \\
\hline Coisas que podem ser usadas para achatar uma folha de videira sem a estragar & 42 & 4.52 \\
\hline Coisas que podem ser usadas para estragar um computador & 44 & 5.27 \\
\hline Coisas construídas por humanos (a) & 30 & 7.97 \\
\hline
\end{tabular}


Bebidas usadas em cocktails exóticos

Bebidas que normalmente são consumidas em mistura com outros ingredientes

Profissões adequadas para quem gosta de viajar

Profissões nas quais o profissional tem mais hipóteses de sujar sua roupa/uniforme

Vegetais que se comem crus

Vegetais para esconder o sabor de outros alimentos

Vegetais que podem ser usados para abanar contra o rosto em clima quente

Animais que podem ser usados para assustar os outros

Animais que podem ser usados para lutar com humanos num ringue

Animais que podem ser ouvidos em áreas montanhosas

Frutas que podem ser usadas para jogar ao berlinde

Frutas para atirar aos outros

Frutas indicadas para acompanhar comidas salgadas

Objeto de cozinha que podem ser usados como armas de tortura

Objetos de cozinha que podem ser usados para caçar uma mosca

\section{Taxonómicas}

$\begin{array}{lll}\text { Coisas de comer } & 69 & 7.28 \\ \text { Roupas } & 49 & 8.51 \\ \text { Desportos } & 48 & 5.44 \\ \text { Instrumentos musicais } & 55 & 8.16 \\ \text { Bebidas } & 62 & 4.98 \\ \text { Profissões } & 63 & 6.54 \\ \text { Vegetais } & 65 & 5.23 \\ \text { Animais } & 60 & 6.15 \\ \text { Frutas } & 69 & 7.67 \\ \text { Objetos de cozinha } & 64 & 6.66\end{array}$

Objetos de cozinha

Nota. (a) Lista originalmente usada em Barsalou (1983). (b) Lista originalmente usada em Barsalou (1985). (c) Lista originalmente usada em Valleé-Torangeau, Anthony \& Austen (1998).

As categorias taxonómicas tendem a evocar mais produções $(M=6,66)$ do que as demais categorias. Categorias ad hoc inter-categóricas, por sua vez, evocam mais produções do que ad hoc intracategóricas ( $M=5,63$ e $M=4,00$ respetivamente).

\section{Procedimento}

As normas foram recolhidas através de 11 questionários diferentes respondidos em papel e lápis e em computador. Destes, quatro eram compostos por categorias taxonómicas e ad hoc intra-categóricas sendo que as categorias que apareciam em versão taxonómica num questionário apareciam em versão ad hoc no outro. Dois questionários eram compostos por categorias taxonómicas, ad hoc intra-categóricas e inter- 
categóricas com o mesmo controlo (i.e., cada lista intra-categórica surge como taxonómica ou como ad hoc em cada questionário). Três questionários eram compostos apenas por categorias ad hoc intercategóricas, e dois apenas por categorias ad hoc intra-categóricas. Os questionários tinham de oito a dez categorias e foram respondidos por alunos de psicologia em sala de aula. Os questionários em computador foram respondidos num laboratório experimental. Dos 425 questionários respondidos, 321 foram em papel e lápis e 104 foram em computador. Nas instruções era pedido que os participantes escrevessem exemplares para as categorias apresentadas, dando preferência a exemplares que lhes viessem imediatamente à mente e tentando nomeá-los usando apenas uma palavra. Contudo, não houve limite de respostas para o número de exemplares gerados. Dado que as categorias ad hoc fogem ao formato das categorias taxonómicas, foi incluído nas instruções um exemplo de categoria ad hoc com alguns exemplares (sempre não relacionado com as categorias a responder seguidamente pelos participantes). Categorias ad hoc intra-categóricas não foram apresentadas no mesmo questionário que as categorias taxonómicas de onde estas foram derivadas.

\section{RESULTADOS}

As listas obtidas e as suas informações associadas estão disponíveis como material suplementar. As listas de exemplares para cada categoria foram ordenadas por frequência de produção. A primeira coluna de resultados (Menções) indica a quantidade absoluta de participantes que mencionaram o exemplo; a segunda coluna (Frequência de produção) indica a quantidade relativa de respostas do exemplar em função da quantidade de participantes; a terceira coluna (Classificação) indica o ranking médio da ordem na qual o item foi mencionado, obtido através da soma das posições dos exemplares na ordem de produção dividida pela quantidade total de produções; e a quarta coluna (Primeiro) indica a proporção com que o exemplar foi produzido em primeiro lugar, obtida através da soma de participantes que produziram o exemplar em primeiro lugar dividido pelo número de participantes que responderam à categoria.

Exemplares com erros gramaticais foram alterados para a sua forma correta. Os exemplares foram transformados para a sua versão no singular. Quando havia produções do exemplar no plural foi incluída a sua terminação plural em parênteses ao lado da palavra no singular. Alguns exemplares foram agregados por ter sido julgado que representavam a mesma ideia dentro de uma categoria (e.g., instâncias de "Madeira" e "Lenha" foram agregadas no item "Madeira" na categoria "Coisas inflamáveis"). Apesar disso, os resultados relativos a estas variações são também apresentados caso seja do interesse do investigador, de modo que os exemplares possam ser agregados de outras formas que o mesmo achar mais adequado. Exemplares ininteligíveis nos questionários de papel e lápis (menos de $1 \%$ do total) foram eliminados. Em alguns casos, os exemplares criados não pertenciam à categoria, mas sim provinham de associação livre à categoria (e.g., respostas como "vida", "selvagem" e "doméstico" na categoria "animais") e nestes casos todos os exemplares produzidos para a categoria foram eliminados da amostra. Em alguns casos o participante foi eliminado da amostra por ter respondido desta maneira a todas as categorias ( 4 participantes no total), e noutros casos foram eliminadas apenas suas respostas para a categoria em questão (.016 do total de categorias respondidas por todos os participantes).

A média de frequência de produção (dos exemplares com mais frequência de produção) das listas taxonómicas $(M=.79)$ é maior do que os demais tipos de categorias, sendo a sua variação menor $(D P=$ $.10)$. As categorias ad hoc inter $(M=.71, D P=.18)$ e intra-categóricas $(M=.65, D P=.18)$ apresentam valores médios e de dispersão semelhantes. A diferença de variação fica aparente quando comparamos valores máximos e mínimos de exemplares com mais frequência de produção em categorias taxonómicas (.65 e .92), ad hoc inter-categóricas (.34 e 1.00) e ad hoc intra-categóricas (.35 e 1.00).

\section{DISCUSSÃo}

As medidas normativas de exemplares de categorias taxonómicas constituem um material valioso para a investigação sobre representações mentais de categorias, permitindo explorar como essas representações tendem a ser organizadas (e as características dos exemplares normalmente encontrados em diversos níveis da dimensão de tipicidade) e como estas representações de categorias são acedidas para utilização noutros processos cognitivos. 0 objetivo deste artigo é disponibilizar medidas normativas para categorias $a d$ hoc, pois possibilitam explorar estruturas hierárquicas categóricas que, embora se organizem como categorias taxonómicas, encerram duas importantes diferenças: 0 facto de não possuírem representações estruturadas pré-existentes e o facto de frequentemente serem orientadas para fins específicos. Contudo, convém notar que algumas categorias ad hoc usadas não estão 
necessariamente ligadas a objetivos (e.g., "Coisas inflamáveis"). Nestes casos, a ligação explícita a objetivos ficará a cargo do investigador, em função dos seus intuitos experimentais. Outro fator a ser levado em conta são as potenciais diferenças individuais entre participantes que possibilitam a existência de casos onde uma categoria, que se assume ser ad hoc, tenha sido alvo de considerável e repetida ativação por parte de um indivíduo, fazendo com que possua estrutura e organização pré-existentes.

Estudos recentes têm feito uso de categorias ad hoc de forma a explorar a natureza maleável, dinâmica e dependente do contexto que as representações mentais destas categorias apresentam por comparação com as representações e padrões de processamento tipicamente inferidos de estudos realizados com categorias taxonómicas mais comuns. Sem querermos ser exaustivos, seguidamente apresentamos e discutimos brevemente alguns trabalhos representativos da investigação envolvendo categorias ad hoc.

Abdel Rahman e Melinger (2011) investigaram e reportaram interferência semântica em tarefas de nomeação de imagens usando grupos de imagens de palavras pertencentes a diferentes categorias pertencendo, porém, à mesma categoria ad hoc. Este efeito de interferência foi menor do que o normalmente encontrado para palavras pertencentes a uma mesma categoria taxonómica e mostrou-se dependente da apresentação do tema ad hoc. A competição de ativação lexical de grupos semânticos é assim um processo dinâmico e condicionado por contexto.

Soro, Ferreira, Semin, Mata e Carneiro (2017) mostraram e tentaram explicar a ocorrência de falsos reconhecimentos para itens pertencentes a categorias ad hoc não incluídos na apresentação das categorias ad hoc relevantes. Este efeito foi observado com uma menor frequência do que falsos reconhecimentos normalmente encontrados para categorias taxonómicas, porém, ao contrário do efeito de interferência semântica, ele ocorre independente da apresentação do tema das categorias, sugerindo alta flexibilidade contextual na produção e inclusão de exemplares na representação categórica.

Baseando-se em estudos anteriores que mostram que o processamento de categorias taxonómicas tende a ser relacional e o de categorias ad hoc tende a ser específico, Grimaldi, Poston e Karpicke (2015) usaram estas diferenças de processamento entre os dois tipos de categoria para identificar a prevalência de tipos de processamento (relacional ou específico) envolvidos em diferentes tarefas de aprendizagem (especificamente em tarefas de mapeamento conceptual).

Outro possível uso de categorias ad hoc está relacionado com a sua dependência do contexto e ligação com objetivos. Teorias sobre compreensão e construção de metáforas defendem a ideia de que as metáforas se organizam como categorias ad hoc, onde o termo de comparação define as características da categoria e é, ao mesmo tempo, o seu exemplar prototípico. Por exemplo: "o meu trabalho é uma prisão". A categoria ad hoc é composta por exemplares que têm como característica limitarem a liberdade de uma pessoa. Neste sentido, "prisão" é o exemplar prototípico e "meu trabalho" é outro exemplar que ao ser incluído na mesma categoria que "prisão" tem essas características de limite de liberdade salientadas, levando à compreensão da metáfora. Com base nesta teorização, Terai e Nagakawa (2012) utilizaram categorias ad hoc como material na construção de um modelo computacional de compreensão de metáforas.

Os exemplos supracitados tornam evidentes a importância das categorias ad hoc para a investigação e as suas variadas aplicações em psicologia experimental. Com o presente trabalho e com a divulgação destas normas esperamos contribuir para que os investigadores que trabalham em Portugal, noutros Países de Língua Portuguesa ou com participantes cuja língua é o português, possam, cada vez mais, participar ativamente no desenvolvimento futuro de estas e de outras áreas de investigação relacionadas.

\section{Referências}

Abdel Rahman, R., \& Melinger, A. (2011). The dynamic microstructure of speech production: Semantic interference built on the fly. Journal of Experimental Psychology: Learning, Memory, and Cognition, 37, 149-161. http://doi.org/10.1037/a0021208

Barsalou, L. W. (1983). Ad hoc categories. Memory \& Cognition, 11, 211-227. http://doi.org/10.3758/BF03196968

Barsalou, L. W. (1985). Ideals, central tendency, and frequency of instantiation as determinants of graded structure in categories. Journal of Experimental Psychology: Learning, Memory, and Cognition, 11, 629-654. http://doi.org/10.1037/0278-7393.11.1-4.629

Barsalou, L. W. (1991). Deriving categories to achieve goals. In: G. H. Bower (Ed.), The psychology of learning and motivation: Advances in research and theory (pp. 1-64). San Diego, Estados Unidos: Academic Press. http://doi.org/10.1016/s0079-7421(08)60120-6

Barsalou, L. W. (1999). Perceptual symbols systems. Behavioral and Brain Sciences, 22, 577-660. http://doi.org/10.1017/S0140525X99002149 
Battig, W. F., \& Montague, W. E. (1969) Category norms for verbal items in 56 categories - a replication and extension of Connecticut category norms. Journal of Experimental Psychology, 80, 1-46. http://doi.org/10.1037/h0027577

Bousfield, W. A., Cohen, B. H., \& Whitmarsh, G. A. (1958). Associative clustering in the recall of words of different taxonomic frequencies of occurrence. Psychological Reports, 4, 39-44. http://doi.org/10.2466/pr0.1958.4.g.39

Casasanto, D., \& Lupyan, G. (2015). All Concepts are ad hoc concepts. In: E. Margolis \& S. Laurence (Eds.) The Conceptual Mind: New directions in the study of concepts (pp. 543-566). Cambridge, Estados Unidos: MIT Press.

Casey, P. J. (1992). A reexamination of the roles of typicality and category dominance in verifying category membership. Journal of Experimental Psychology: Learning, Memory and Cognition, 18, 823-834. http://doi.org/10.1037//0278-7393.18.4.823

Collins, A. M., \& Loftus, E. F. (1975). Spreading activation theory of semantic processing. Psychological Review, 82, 407-428. http://doi.org/10.1037/0033-295X.82.6.407

Grimaldi, P. J., Poston, P., \& Karpicke, J. D. (2015). How does creating a concept map affect item-specific encoding? Journal of Experimental Psychology: Learning, Memory, and Cognition, 41, 1049-1061. http://doi.org/10.1037/xlm0000076

Hampton, J. A. (1997). Associative and similarity-based processes in categorization decisions. Memory \& Cognition, 25, 625-640. http://doi.org/10.3758/BF03211304

Hampton, J. A., \& Gardiner, M. M. (1983). Measures of internal category structure - A correlational analysis of normative data. British Journal of Psychology, 74, 491-516. http://doi.org/10.1111/j.2044-8295.1983.tb01882.x

Keller, D., \& Kellas, G. (1978) Typicality as a dimension of encoding. Journal of Experimental Psychology: Human Learning and Memory, 4, 78-85. http://doi.org/10.1037//0278-7393.4.1.78

Loftus, E. F., (1973). Category dominance, instance dominance and categorization time. Journal of Experimental Psychology, 97(1), 70-74. http://doi.org/10.1037/h0033782

McCloskey, M. E., \& Glucksberg, S. (1978). Natural categories: well defined or fuzzy sets? Memory \& Cognition, 6, 462-472. http://doi.org/10.3758/BF03197480

Medin, D. L., \& Schaffer, M. M. (1978). Context theory of classification learning. Psychological Review, 85, 207-238. http://doi.org/10.1037//0033-295X.85.3.207

Mervis, M. E., Catlin, J., \& Rosch, E. (1976). Relationships among goodness-of-example, category norms, and word-frequency. Bulletin of the Psychonomic Society, 7, 283-284. http://doi.org/10.3758/BF03337190

Murphy, G. L. (1988). Comprehending complex concepts. Cognitive Science, 12, 529-562. http://doi.org/10.1207/s15516709cog1204_2

Puff, C. R., (1970). Role of clustering in free recall. Journal of Experimental Psychology, 86, 384-386. http://dx.doi.org/10.1037/h0030189

Rips, L. J., (1975). Inductive judgments about natural categories. Journal of Verbal Learning \& Verbal Behavior, 14, 251-269. https://doi.org/10.1016/S0022-5371(75)80055-7

Rosch, E. (1973) Natural categories. Cognitive Psychology, 4, 328-350. http://doi.org/10.1016/00100285(73)90017-0

Rosch, E., \& Mervis, C. B. (1975). Family resemblances: Studies in the internal structure of categories. Cognitive Psychology, 7, 573-605. https://doi.org/10.1016/0010-0285(75)90024-9

Rosch, E., Simpson, C., \& Miller, R. S., (1976) Structural bases of typicality effects. Journal of Experimental Psychology: Human Perception and Performance, 2, 491-502. http://doi.org/10.1037/00961523.2.4.491

Smith, S. M., Ward, T. B., Tindell, D. R., Sifonis, C. M., \& Wilkenfeld, M. J. (2000). Category structure and created memories. Memory \& Cognition, 28, 386-395. http://doi.org/10.3758/bf03198554

Soro, J. C., Ferreira, M. B., Semin, G.R., Mata, A., \& Carneiro, P. (2017). Ad hoc categories and false memories: Memory illusions for categories created on-the-spot. Journal of Experimental Psychology: Learning, Memory, and Cognition. Advance online publication. http://doi.org/10.1037/xlm0000401

Terai, A., \& Nakagawa, M. (2012). A corpus-based computational model of metaphor understanding consisting of two processes. Cognitive Systems Research, 19-20, 30-38. http://doi.org/10.1016/j.cogsys.2012.03.001

Vallée-Tourangeau, F., Anthony, S. H., \& Austin, N. G. (1998). Strategies for generating multiple instances of common and ad hoc categories. Memory, 6, 555-592. http://doi.org/10.1080/741943085 
Van Overschelde, J. P., Rawson, K. A., \& Dunlosky, J. (2004). Category norms: An updated and expanded version of the Battig and Montague (1969) norms. Journal of Memory and Language, 50, 289-335. http://doi.org/10.1016/j.jml.2003.10.003

Ward, T. B., \& Wickes, K. N. S. (2009) Stable and dynamic properties of category structure guide imaginative thought. Creativity Research Journal, 21, 15-23. http://doi.org/10.1080/10400410802633376

Wilkins, A. J. (1971). Conjoint frequency, category size and categorization time. Journal of Verbal Learning \& Verbal Behavior, 10, 382-385. http://doi.org/10.1016/S0022-5371(71)80036-1

Wisniewski, E. J. (1997). When concepts combine. Psychonomic Bulletin \& Review, 4, 167-183. http://doi.org/10.3758/BF03209392

Historial do artigo

Recebido 21/02/2017

Aceite $\quad 18 / 05 / 2017$

Publicado $\quad 07 / 2017$ 